\title{
Determinantes de caries temprana de la infancia en niños en riesgo social.
}

\section{Determinants of early childhood caries in children at social risk.}

\author{
Sonia Echeverria-López ${ }^{1}$, Eugenia Henríquez-D’Aquino ${ }^{1 *}$, Fabiola Werlinger-Cruces ${ }^{2}$, \\ Tania Villarroel-Díaz ${ }^{3}$, Mónica Lanas-Soza ${ }^{3}$.
}

\section{Departamento del Niño y Ortopedia} DentoMaxilar, Facultad de Odontología, Universidad de Chile, Santiago, Chile. 2. Centro de Epidemiología y Vigilancia de las Enfermedades Orales (CEVEO). Facultad de Odontología. Universidad de Chile, Santiago, Chile. 3. Cirujano Dentista.. Práctica Privada, Santiago, Chile.

* Correspondencia Autor: Eugenia HenríquezD’Aquino | Dirección: Olivos 943, Independencia, Santiago, Chile | Teléfono: +562 2978 1725 |

E-mail: eugeniahd@gmail.com

Trabajo recibido el 01/12/2019.

Aprobado para su publicación el 30/12/2019

\section{RESUMEN}

Objetivo: Determinar la prevalencia de caries temprana de la infancia en niños en riesgo social y analizar sus factores de riesgo asociados. Método: Se realizó un estudio descriptivo de corte transversal con 246 niños de 24 a 71 meses de edad, reclutados de 13 barrios marginales, en Santiago, Chile. Se utilizó un cuestionario para obtener información sobre etnia, peso al nacer, edad y educación de la madre, uso de biberón nocturno, cepillado de dientes y visitas dentales. La caries temprana de la infancia fue registrada de acuerdo a la definición aceptada por la Academia Americana de Pediatría Dental. Se utilizó un análisis de regresión logística múltiple para investigar la influencia de los factores de riesgo en la experiencia de caries. Resultados: La prevalencia de caries temprana de la infancia fue de un $63 \%$. Los análisis bivariados mostraron asociaciones entre etnicidad, educación de la madre, uso de biberón nocturno, visitas dentales y caries temprana de infancia. El modelo multivariado final, mostró que los niños cuyas madres tenían un bajo nivel de educación tenían mayores probabilidades de desarrollar caries temprana de la infancia. Conclusión: Los niños estudiados tuvieron una alta prevalencia de caries temprana de la infancia, siendo la educación de la madre, el determinante más importante.

PALABRAS CLAVE

Caries temprana de la infancia; Prevalencia; Determinantes, Riesgo social, Factores de riesgo.

International Journal of Interdisciplinary Dentistry Vol. 13(1); 26-29, 2020.

\section{ABSTRACT}

Objective: The aim of the present study was to evaluate the prevalence of early childhood caries (ECC) among children at social risk and to analyze its associated determinants. Method: A cross-sectional study with 246 children aged 24 to 71 months, recruited from 13 different slums, below the poverty line, in Santiago, Chile was performed. An interviewer-administered questionnaire was used to obtain information from the parents on ethnicity, birth weight, mother's age and education, night bottle feeding, tooth brushing and dental visits. Early childhood caries was defined using the American Academic of Pediatric Dentistry criteria. Multiple logistic regression analysis with a stepwise selection procedure was used to investigate the influence of risk factors on the early childhood caries experience. Results: The prevalence of early childhood caries was $63 \%$. Bivariate analyses showed associations among ethnicity, mother's education, bottle feeding at night, dental visits and caries experience. The final multivariate model showed that children whose mothers had a low level of education were more likely to develop early childhood caries. Conclusions: The children at social risk studied had a high prevalence of early childhood caries, with the education of the mother as the most important determinant.

\section{KEY WORDS}

Early childhood caries; Prevalence; Determinants; Social risk, Risks factors.

International Journal of Interdisciplinary Dentistry Vol. 13(1); 26-29, 2020.

\section{INTRODUCCIÓN}

La caries temprana de la infancia $(\mathrm{CTI})$ es reconocida como un problema de salud pública debido a su alta prevalencia y su impacto en la salud, en el desarrollo y la calidad de vida de los niños que la padecen ${ }^{(1,2)}$. Es una enfermedad crónica, no contagiosa y prevenible ${ }^{(2)}$, considerada de etiología compleja y multifactorial|(3), afecta a párvulos, siendo más prevalente en los grupos en riesgo social, especialmente entre niños que viven en situación de pobreza, migrantes o refugiados ${ }^{(4,5,6,7)}$. Factores de riesgo tales como etnicidad, ingreso familiar, nivel educacional de la madre, nivel socioeconómico familiar, conocimiento de los padres en relación a salud oral, creencias y prácticas alimentarias han sido 
asociados con $\left.\mathrm{CT}\right|^{(8,9,10)}$. Además, se ha observado que otros factores como un bajo peso al nacer, la presencia de defectos del esmalte, junto al uso inapropiado del biberón, sobre todo cuando el niño duerme, tienen un papel importante en la etiología y la severidad de la $C T^{(11,12,13)}$.Varios estudios han demostrado la interacción entre factores socio demográficos, conductuales, biológicos y $\mathrm{CTI}$, donde los factores sociales como la pobreza y el bajo nivel socioeconómico tienen una influencia sustancial en el desarrollo de la enfermedad(4,5,13,14). La pobreza es un determinante social significativo para la salud oral a un nivel global(15). Niños con riesgo social, muestran mayores indicadores de caries y factores de riesgo que niños sin desventaja social(16).

Estos factores no se han abordado bien en los niños en riesgo social, existen pocos datos disponibles que describan la prevalencia y los factores de riesgo de CTI en niños que viven por debajo de la línea de la pobreza. Hasta la fecha, en Chile, aunque varios estudios han examinado la experiencia de CTI entre niños de bajos niveles socioeconómicos, no se ha realizado ningún estudio dentro de una población en condiciones de extrema pobreza. Por lo tanto, el objetivo de este estudio fue determinar la prevalencia de caries temprana de la infancia en niños que viven en riesgo social y señalar sus factores de riesgo asociados.

\section{MATERIAL Y MÉTODO}

Se realizó un estudio observacional de corte transversal en 246 niños de 24 a 71 meses de edad, reclutados de 13 barrios marginales ("campamentos") de la ciudad de Santiago, Chile, aprobado para su realización por el Comité de Ética de la Facultad de Odontología de la Universidad de Chile (Proyecto PRI-ODO 1001). Los niños y sus madres o tutores participaron voluntariamente una vez que fueron contactados e invitados a participar. Se obtuvo el consentimiento informado por escrito de cada participante antes de que los niños fueran examinados.

Para el cálculo del tamaño de la muestra, se consideró una tasa de prevalencia del $30 \%$ para CTI, un intervalo de confianza (IC) del $95 \%$, un error estándar del $6 \%$ y una posible tasa de no respuesta del $10 \%$, que determinó una muestra mínima de 246 niños preescolares de entre 24 y 71 meses de edad. Dadas las dificultades con el acceso a la población de estudio, considerando además su condición de vulnerabilidad, se adoptó un muestreo por conveniencia (no probabilístico) según participación voluntaria. Los datos fueron recolectados con el uso de un instrumento de recolección de datos que contenía 7 variables que cubrían varios aspectos de los niños estudiados, estos incluían: etnia, peso al nacer, edad y educación de la madre, alimentación con biberón nocturno, cepillado dental y visitas dentales. Estas variables estuvieron basadas en estudios previos que mostraron tener una asociación con $\left.\mathrm{CT}\right|^{(3,10,14)}$. El instrumento se administró en una entrevista cara a cara para obtener información y se piloteó previamente para determinar la claridad de las preguntas en 20 madres o tutores de niños de entre 24 y 71 meses, que provenían de los mismos barrios participantes en el estudio, pero que no formaron parte del mismo. Antes de comenzar el trabajo de campo, se realizó un ejercicio de entrenamiento y calibración para el diagnóstico de CTI, el cual se realizó utilizando imágenes clínicas de CTI y la calibración se llevó a cabo en la Clínica de Odontopediatría de la Facultad de Odontología de la Universidad de Chile, con un examen oral de 20 párvulos, en dos ocasiones separadas con un intervalo de una semana entre sesiones. Los valores de concordancia obtenidos (índice Kappa) fueron altos alcanzado 0.86 para el acuerdo inter examinador y 0.92 para el acuerdo intra examinador.

Se realizó un examen clínico oral a los niños, con 2 examinadores calibrados para CTI, registrándose el índice ceod y ceos de acuerdo con los criterios recomendados por la Organización Mundial de la Salud, donde el componente "c" incluyó las lesiones cavitadas y no cavitadas de caries.

Descripción de las variables: Las variables estudiadas se definieron de la siguiente manera: la presencia de CTI fue determinada, de acuerdo a la definición aceptada por la Academia Americana de Pediatría Dental (AAPD), como la presencia de una o más superficies dentarias cariadas (con lesiones cavitadas o no cavitadas), perdidas por caries u obturadas en cualquier diente primario, en niños menores de 6 años de edad ${ }^{(17)}$. La etnicidad se definió por el auto informe del participante de pertenecer a un grupo étnico. El peso al nacer se dividió en menos de 2.500 gramos y 2.500 gramos o más. La edad materna se dividió en dos grupos: madres menores de 24 años y madres mayores de 24 años. La educación de la madre se dividió en 2 categorías: (1) madres con menos de 12 años de educación y (2) madres con más de 12 años de educación. La alimentación con biberón nocturno fue dicotomizado en sí / no. Las visitas dentales se dividieron en 2 grupos de edad: a los 2 años y 4 años y se caracterizó en asistidos / no asistidos. La frecuencia de cepillado dental se clasificó como "menos de dos veces al día" y "más de dos veces al día".
Análisis estadístico: El análisis de los datos se realizó con el software Stata, versión $11.0^{\circledR}$ (StataCorp LP, College Station, Texas, EE. UU.). Los resultados se presentan como la prevalencia de CTI y experiencia de caries (ceod y ceos), así como CTI y factores asociados. El análisis univariado se realizó mediante el cálculo de estadísticas descriptivas según la escala de medición de cada variable (media, desviación estándar y frecuencia relativa). Para comparar la experiencia de caries por sexo y edad (análisis bivarido), se utilizó la prueba de Chi Cuadrado (prevalencia de caries); y las pruebas T de Student, Wilcoxon no pareado, ANOVA y Kruskal-Wallis (ceod y ceos). En el análisis multivariado se aplicó un modelo de regresión logística paso a paso hacia atrás (stepwise backward) con la estimación de medidas de diparidad (odds ratio) e intervalos de confianza del 95\%. El nivel de significación estadística se fijó en un valor de $\alpha<0,05$.

\section{RESULTADOS}

La muestra estuvo constituida por 246 párvulos de entre 24 y 71 meses. La edad promedio de los niños fue de 48,3 $\pm 14,07$ meses. Un $46.3 \%$ de los niños fueron hombres y un $57.3 \%$ de niñas. Mientras que solo alrededor del $10 \%$ de los niños que participaron pertenecían a algún grupo étnico. La figura 1, muestra la distribución de niños en los diferentes barrios marginales de la ciudad de Santiago con la prevalencia de CTI y sus respectivas tasas de ceod encontradas en cada uno de ellos.

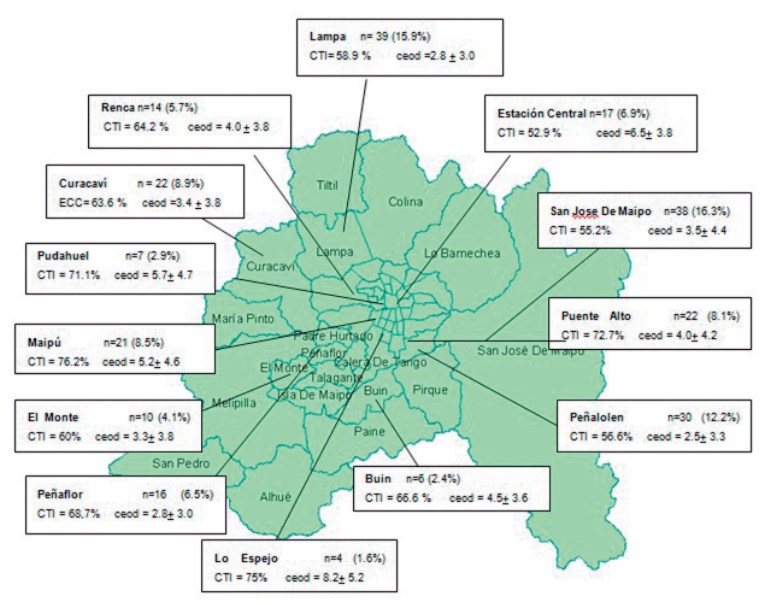

Figura 1. Distribución, prevalencia de caries temprana de la infancia (CTI) e índice de ceod de niños que viven en extrema pobreza en Santiago, Chile.

La prevalencia de caries fue de un 63\% [IC95\%: 56,2 - 68,7\%], mientras que, el valor promedio ceod obtenido en este estudio fue de 3,5 $+4,02$ [IC95\%: 3,0 - 4.0]. La experiencia de caries en los niños estudiados según sexo y edad se representa en la Tabla 1. La prevalencia de caries en los cuatro grupos de edad mostró una diferencia significativa ( $p=$ 0.001 ), mostrando una mayor prevalencia a medida que aumenta la edad de los niños.

\section{Caries temprana de la infancia y Factores de riesgo asociados.}

Se realizó un análisis bivariado que mostró asociación entre CTI y los factores de etnia $(p=0,031)$, educación de la madre $(p=0,002)$, alimentación con biberón nocturno $(p=0,001)$ y control dental a los 2 años $(p=0,025)$. Los factores restantes (Tabla 2), no fueron estadísticamente significativos en este análisis ( $p>0.05)$.

En el análisis de regresión logística múltiple paso a paso hacia atrás, a partir de un modelo completo, se identificó que los factores de riesgo significativos fueron: educación de la madre, alimentación con biberón nocturno y control dental a los 2 años de edad. El modelo multivariado (Tabla 3) final mostró que solamente los niños cuyas madres tenían un bajo nivel de educación tenían más probabilidad de desarrollar caries temprana de la infancia $(O R=2.51, \mathrm{IC} 95 \%: 1.42-4.44, \mathrm{P}=0.002)$. Mientras que la alimentación con biberón nocturno y una visita al dentista a los 2 años de edad, mostraron efectos en la estimación de la asociación esperada en la dirección opuesta, sin encontrar asociación entre la etnia y CTI.

\section{DISCUSIÓN}

El presente estudio proporciona evidencia sobre los determinantes de 
Tabla 1: Experiencia de caries según sexo y edad de la muestra.

\begin{tabular}{|c|c|c|c|c|c|}
\hline \multirow{3}{*}{ Edad } & $24-35$ & $63(25.6)$ & $24(38.1)$ & $1.25+2.411$ & $1.68+3.901$ \\
\hline & $48-59$ & $56(22.8)$ & $38(67.9)$ & $4.33+4.283$ & $6.76+8.133$ \\
\hline & $60-71$ & $75(30.5)$ & $63(84.0)$ & $5.30+4.101,3$ & $9.18+10.721,3$ \\
\hline Sexo & Mujer & $132(53.7)$ & $86(60.6)$ & $3.43+3.89$ & $5.18+6.98$ \\
\hline Valor $\mathrm{P}$ & & & $0.486 a$ & $0.488 d$ & $0.704 \mathrm{e}$ \\
\hline Total & & $246(100)$ & $154(62.6)$ & $3.54+4.02$ & $5.59+8.35$ \\
\hline
\end{tabular}

1 Diferencias estadísticamente significativas con grupo de 36-47 meses

2 Diferencias estadísticamente significativas con grupo de 48-59 meses

3 Diferencias estadísticamente significativas con grupo de 24-35 meses

SD: Desviación Estándar; Prom: Promedio

a Prueba de Chi Cuadrado; b Prueba ANOVA;

c Prueba de Kruskal Wallis; d Prueba T de Student no pareada;

e Prueba de Wilcoxon no pareado

Tabla 2: Asociación entre factores de riesgo y caries temprana de la infancia. Análisis bivariado

\begin{tabular}{|c|c|c|c|}
\hline Variables & $\mathrm{n}(\%)$ & OR $(95 \%)$ & Valor $p$ \\
\hline \multicolumn{4}{|l|}{ Etnicidad } \\
\hline $\mathrm{Si}$ & $26(10.6)$. & $0.39(0.16-0.98)$ & $0.024^{*}$ \\
\hline \multicolumn{4}{|l|}{$\mathrm{No}^{\mathrm{a}}$} \\
\hline \multicolumn{4}{|c|}{ Peso al nacer } \\
\hline$>2.500 \mathrm{~g}$ & $28(11.4)$ & $1.92(0.74-5.56)$ & 0.213 \\
\hline \multicolumn{4}{|l|}{$<2.500 \mathrm{~g}^{\mathrm{a}}$} \\
\hline \multicolumn{4}{|c|}{ Nivel educacional de la madre } \\
\hline$>12$ años & $98(34.8)$ & $2.26(1.29-3.99)$ & $0.002^{*}$ \\
\hline \multicolumn{4}{|l|}{$<12$ años ${ }^{a}$} \\
\hline \multicolumn{4}{|c|}{ Edad de la madre } \\
\hline$>24$ años & $93(60.3)$ & $0.88(0.50-1.54)$ & 0.645 \\
\hline \multicolumn{4}{|l|}{$<24$ años ${ }^{a}$} \\
\hline \multicolumn{4}{|c|}{ Biberón nocturno } \\
\hline $\mathrm{Si}$ & $118(48.0)$ & $0.04(0.22-0.71)$ & $0.001^{*}$ \\
\hline \multicolumn{4}{|l|}{$\mathrm{No}^{\mathrm{a}}$} \\
\hline \multicolumn{4}{|c|}{ Control dental a los 2 años } \\
\hline No & $114(46.3)$ & $0.54(0.31-0.94)$ & $0.023^{*}$ \\
\hline \multicolumn{4}{|l|}{$\mathrm{Si}^{\mathrm{a}}$} \\
\hline \multicolumn{4}{|c|}{ Control dental a los 4 años } \\
\hline No & $51(38.9)$ & $0.94(0.38-2.39)$ & 1.000 \\
\hline \multicolumn{4}{|l|}{$\mathrm{Si}^{\mathrm{a}}$} \\
\hline \multicolumn{4}{|c|}{ Frecuencia de cepillado } \\
\hline$>2$ al día & $88(35.8)$ & $1.45(0.82-2.64)$ & 0.177 \\
\hline \multicolumn{4}{|l|}{$<2=$ al día ${ }^{a}$} \\
\hline \multicolumn{4}{|c|}{$\begin{array}{l}\text { a Grupo de referencia (No expuesto) } \\
\text { *Valor P estadísticamente significativo } \\
\text { Valor P calculado mediante Prueba Chi cuadrado }\end{array}$} \\
\hline
\end{tabular}

caries temprana de la infancia en niños chilenos, que viven en riesgo social. Los resultados obtenidos mostraron una prevalencia de CTI, de un $63 \%$, siendo el factor de riesgo de caries más fuertemente asociado con
Tabla 3: Modelo de regresión final para las variables asociadas con caries temprana de la infancia

\begin{tabular}{|l|c|c|c|}
\hline \multicolumn{1}{|c|}{ Variables } & $\begin{array}{l}\text { Odss } \\
\text { Ratio }\end{array}$ & $\mathbf{9 5 \%} \mathbf{C l}$ & Valor $\mathbf{p}$ \\
\hline $\begin{array}{l}\text { Nivel educacional de la madre } \\
\text { (Ref: >= 12 años) }\end{array}$ & 2.91 & $1.42-4.54$ & 0.012 \\
\hline $\begin{array}{l}\text { Biberón nocturno } \\
\text { (Ref: No) }\end{array}$ & 0.20 & $0.42-0.72$ & $<0.002$ \\
\hline $\begin{array}{l}\text { Control dental a los 2 años } \\
\text { (Ref: Si) }\end{array}$ & 0.31 & $0.29-0.92$ & $<0.067$ \\
\hline
\end{tabular}

el desarrollo de la enfermedad en estos niños, el bajo nivel educacional de la madre. En nuestro país hay muy poca evidencia publicada en relación al tema, con la cual se puedan contrastar estos resultados. En 2007, un estudio llevado a cabo por el Ministerio de Salud de Chile ${ }^{(18)}$, reportó una prevalencia de caries a los 2 años de un $17 \%$ y de un $48 \%$ a los 4 años, en relación a estos valores, los resultados obtenidos en nuestro estudio son más altos para los mismos grupos etarios: $38 \%$ de caries a los 2 años y $68 \%$ de caries a los 4 años de edad. Con respecto a otros estudios publicados en Chile, la prevalencia de CTI encontrada en este estudio, es mayor a la prevalencia reportada por Hoffmeister et al $^{(19)}$, en 2016, estando en concordancia con los resultados obtenidos por Uribe et $\mathrm{al}^{(20)}$, en 2013, y menor a los resultados obtenidos por Zaror et al, en 2011(21).

En relación a los factores de riesgo de caries asociados, los resultados del análisis multivariado indicaron que un bajo nivel de educación de la madre tuvo un impacto significativo en la prevalencia de CTI en estos niños, respaldando los hallazgos de estudios previos, en los que los investigadores identificaron una asociación entre bajo nivel socioeconómico, bajo nivel de educación de la madre y la ocurrencia de $\left.C T\right|^{(5,22,23,24)}$. Las madres con bajos niveles de educación y bajo nivel socioeconómico, tienden a tener un conocimiento deficiente sobre higiene oral(22,23), teniendo más prácticas de alimentación cariogénicas y más ansiedad con creencias negativas y actitudes hacia el cuidado dental $^{(25)}$. Las madres están usualmente, más involucradas en el cuidado de los niños durante los primeros 5 años de vida, lo que indicaría que un mayor nivel educacional de la madre se asocia a mejores patrones de cuidado infantil. Otros factores que podrían estar asociados, tales como autoestima y síntomas depresivos, podrían explicar el hecho de que las madres ofrezcan una mayor cantidad de alimentos cariogénicos a sus hijos ${ }^{(25)}$. Con respecto a los otros factores involucrados en este estudio, el control dental a los 2 años y la alimentación con biberón por la noche, mostraron resultados contradictorios, si bien, el análisis bivariado mostró que estos factores estaban relacionados con la CTI, en el análisis multivariado, ambos fueron factores protectores contra la CTI. Este hallazgo puede ser contradictorio y su explicación puede estar 
relacionada, en el caso de la alimentación con biberón, a un sesgo de cortesía, donde los padres o tutores respondieron lo que ellos creían sería aprobado. Con respecto al control dental, puede deberse a un sesgo de información debido a que muchos padres llevan a sus hijos a los servicios de salud solo en caso de emergencia para el tratamiento del dolor, sobre todo a menores edades, lo cual no fue discriminado por el cuestionario, el cual solo preguntaba si asistió o no al dentista sin consultar específicamente la causa de esa visita.

La falta de atención dental adecuada ha sido otro problema importante para los niños que viven en situación de pobreza ${ }^{(3)}$, las visitas dentales antes de los 2 años de edad son importantes para mantener a los niños pequeños libres de caries ${ }^{(3)}$. En este estudio, el $46.3 \%$ de los niños nunca habían ido al dentista a los 2 años de edad y presentaron una alta prevalencia de CTI a esa edad $(70.2 \%)$, es probable que este hallazgo se asocie con los bajos niveles de educación de sus madres que desconocían la importancia de esta medida y además porque las condiciones socioeconómicas afectan el conocimiento de salud bucal de los padres ${ }^{(5,16)}$. Los factores que podrían haber contribuido al acceso inadecuado a la atención dental de estos niños, incluyen la ubicación geográfica de los barrios marginales y la falta de transporte adecuado para llegar a los centros de salud, junto al hecho de que muchos de estos hogares están encabezados por mujeres, que dejan a sus hijos solos o al cuidado de otros, al momento de ir a trabajar.

Las condiciones de pobreza han sido identificadas como un factor de riesgo significativo para caries temprana de la infancia. La educación inadecuada puede considerarse una forma de pobreza, por lo tanto, se podría argumentar que el efecto de la educación materna sobre la prevalencia de CTI observada en esta investigación es el resultado de las condiciones de pobreza. Aunque la pobreza no se definió como uno de los factores de riesgo de CTI en este estudio, se puede obtener evidencia indirecta de ello al encontrar que un nivel de educación materna más bajo tenía una mayor posibilidad de desarrollar caries temprana de la infancia, y este factor se mantuvo asociado con CTI, incluso hasta el final del análisis multivariado.

Otro punto importante de este trabajo, es el hecho, de que los resultados muestran una alta prevalencia de caries en edades muy tempranas, a los 2 años se encontró que los niños ya presentaban por lo menos entre 2 y 4 superficies cariadas con una alta prevalencia de CTI, de alrededor de un $40 \%$. Esta condición es preocupante, ya que en los niños pequeños, los tratamientos son complejos y de alto costo cuando la magnitud del daño es muy severa, lo cual se incrementa con la edad.

Este estudio tiene una ventaja entre los estudios de CTI, ya que incorpora niños que no son comúnmente estudiados o representados en estudios demográficos relacionados con el estado socioeconómico y CTI, la investigación se llevó a cabo completamente dentro de una población de niños en riesgo social, de diferentes barrios marginales, ubicados en las afueras de la ciudad de Santiago, Chile. Esto constituye la principa fortaleza de esta investigación debido a las dificultades encontradas durante el proceso de organización relacionadas con el acceso y el contacto con la población en estudio, donde muy pocos estudios han investigado una población con estas características ${ }^{(16)}$.

Finalmente, se deben abordar algunas limitaciones potenciales de este estudio, y aunque, este estudio proporciona evidencia de la influencia de la pobreza en la prevalencia de CTI, es un estudio transversal, con una muestra seleccionada por conveniencia. Se necesitan más estudios para evaluar los efectos a largo plazo de las condiciones de pobreza en la experiencia de caries de estos niños, donde además de los factores conductuales y socio demográficos, sería interesante identificar otros factores que actúen en el desarrollo de la enfermedad en esta población. Otro punto a mencionar, es el tamaño de la muestra, si bien, la muestra se reclutó en un área geográfica amplia, la muestra fue relativamente pequeña debido a las dificultades para acceder a la población de estudio, por lo tanto, los resultados solo se pueden aplicar a áreas de similares condiciones.

En conclusión, los niños en riesgo social estudiados tuvieron una alta prevalencia de caries temprana de la infancia, siendo la educación de la madre, el determinante más importante. Estos resultados muestran que la promoción de la salud oral y los programas preventivos no han sido del todo efectivos para prevenir el desarrollo de CTI en esta población de niños que vive en la marginalidad. Además, estos resultados sugieren que las estrategias preventivas para CTI deben prestar atención no solo al niño sino también al conocimiento y comportamiento materno. Un cambio en la conducta de la madre puede representar una parte importante de la prevención de caries temprana de la infancia en niños en riesgo social, la cual, en esta población desfavorecida, debe centrarse en la educación de las madres y el examen dental precoz y oportuno de los niños, en sus primeras edades.

\section{CONCLUSIÓN}

Los niños estudiados tuvieron una alta prevalencia de caries temprana de la infancia, siendo la educación de la madre, el determinante más importante.

\section{CONFLICTO DE INTERÉS}

Los autores del trabajo declaran no tener ningún conflicto de intereses.

\section{Bibliografía}

1.Özen B, Van Strijp A, Özer L, Olmus H, Genc A, Burcak S. Evaluation of possible associated factors for early childhood caries and severe early childhood caries: A multicenter cross-sectional survey. J Clin Pediatr Dent. 2016;40(2):118-123.

2. Begzati A, Berisha M, Meqa K. Early childhood caries in preschool children of Kosovo - a serious public health problem. BMC Public Health 2010;10:788.

3. Sukurmaran A, Pradeep A. Early childhood caries: Prevalence, risk factors, and prevention. Front Pediatr. 2017:5(7):1-8.

4. Baggio S, Abarca M, Bodenmann P, Gehri M, Madrid C. Early childhood caries in Switzerland: A marker of social inequalities. BMC Oral Health. 2015;15(1):82 5. Van der Tas J, Kragt L, Elfrink M, Bertens L, Jaddoe V, Moll H Et al. Socia inequalities and dental caries in six-year-old children from the Netherlands. J Dent. 2017;62:18-24

6. Peltzer K, Mongkolchati A. Severe early childhood caries and social determinants in three-year-old children from northern Thailand: A birth cohort study. BMC Ora Health. 2015;15:108

7. Naidu R, Nunn J, Kelly A. Socio-behavioural factors and early childhood caries: a cross-sectional study of preschool children in central Trinidad. BMC Oral Health. 2013,13:30

8. Alshehri A. Social and behavioral determinants of early childhood caries in the Aseer Region of Saudi Arabia. Ped Dent Care. 2016,1:114:1-5

9. Jain M, Namdev R, Bodh M, Dutta S, Singhal P, Kumar A. Social and behavioral determinants for early childhood caries among preschool children in India. J Dent Res Dent Clin Dent. 2015;9(2):115-120.

10. Fan C, Wang W, Xu T, Zheng C. Risk factors of early childhood caries among children in Beijing - a prospective cohort study. BMC Oral Health. 2019;19:34

11.Jayakumar A, Gurunathan D. Relationship between low birth weight and early childhood caries: A systematic review. International J Pharma and Bio Scienc. 2017;8(3):484-488

12. Paglia L, Scaglioni S, Torchia V, De Cosmi V, Moretti M, Marzo G. Familial and dietary risk factors in early childhood caries. Eur J Paediat Dent. 2016;17(2):93-99. 13. Moimaz S, Carvalho H. Early Childhood Caries: Epidemiology, severity and socio behavioural determinants. Oral Health \& Prev Dent. 2016:14:77-83
14. Sun HB, Zhang W, Zhou XB. Risk factors associated with early childhood caries. Chin J Dent Res. 2017;20(2):97-104

15. Sgan-Cohen HD, Mann J. Health, oral health and poverty. J Am Dent Assoc 2007;138(11):1437-1442.

16. Squassi A, Mauro S, Mauro MJ, Sanchez G, Bordoni N. Relationship between oral health in children and poverty related factors. Acta Odontol Latinoam. 2008;21(1):49-56

17. AAPD: American Academy of Pediatric Dentistry. Policy on early childhood caries (ECC): Classifications, consequences and preventive strategies. Pediatr. Dent. 2010;32:15

18. Ceballos $M$, et al. Diagnóstico en salud bucal de niños de 2 y 4 años que asisten a la educación preescolar. MINSAL Región Metropolitana. 2007. [Consultado 06/08/2019]. Disponible en: http://www.redsalud.gov.cl/archivos/salud_bucal/ PerfilEpi2008.pdf.

19. Hoffmeister L, Moya P, Vidal C, Benadol D. Factors associated with early childhood caries in Chile. Gac Sanit. 2016;30(1):59-62

20. Uribe S, Rodríguez M, Peigna G, Provoste P, Jara L. Prevalencia de caries temprana de la infancia en zona rural del sur de Chile. Ciencia Odontol. 2013;10(2):97-104

21. Zaror C, Pineda P, Orellana J. Prevalencia de caries temprana de la infancia y sus factores asociados en niños chilenos de 2 y 4 años. Int J Odontomat. 2011;5 (2): 171.177

22. Bhardwaj SV, Bhardwaj A. Early childhood caries and its correlation with maternal education level and socio-economic status. J Orofac Sci. 2014;6:53-7

23. Feldens $\mathrm{C}$, Kramer P, Sequeira M, Rodrigues P, Vítolo M. Maternal education is an independent determinant of cariogenic feeding practices in the first year of life. Eur Arch Paediatr Dent. 2012;13(2):70-5

24. Plonka KA, Pukallus ML, Barnett AG, Holcombe TF, Walsh LJ, Seow WK. A longitudinal case control study of caries development from birth to 36 months. Caries Res. 2013; 47:117-127.

25. Abduljalil H, H. Abuaffan A. Knowledge and practice of mothers in relation to dental health of preschool children. Adv Gen Eng. 2016;05(1). 